\title{
Erratum: Spin transfer torque in antiferromagnetic spin valves: From clean to disordered regimes [Phys. Rev. B 89, 174430 (2014)]
}

\author{
Hamed Ben Mohamed Saidaoui, Aurelien Manchon, and Xavier Waintal \\ (Received 5 June 2016; published 29 June 2016)
}

DOI: 10.1103/PhysRevB.93.219906

It was recently brought to our attention that the discussion concerning the current-driven antiferromagnetic dynamics (first paragraph, left column, page 4) in our publication Ref. [1] was incorrect. In this paragraph, we argued that the torque that efficiently manipulates the antiferromagnetic order parameter of a collinear bipartite antiferromagnet is the staggered torque, i.e., the torque that changes sign on the two antiferromagnetically coupled sublattices. This torque, tagged coherent torque in Ref. [1], arises from a uniform, nonstaggered spin density. This statement, consistent with Ref. [2], is actually incorrect. The torque coming from a uniform, nonstaggered spin density results in a spin-flop transition of the antiferromagnet and, therefore, is equally inefficient in changing the antiferromagnetic order parameter direction as a uniform magnetic field. It was argued in Refs. [3-6] that in order to efficiently manipulate the antiferromagnetic order parameter, the spin density itself should be staggered, thereby resulting in a uniform, nonstaggered torque. This is due to the unique dynamics of the antiferromagnetic order parameter, which is driven by the precession of the sublattice magnetic momenta around the antiferromagnetic exchange field [6]. As a consequence, in Ref. [1] and following the terminology used in this paper, the exchange torque is the one that can efficiently manipulate the antiferromagnetic order parameter, while the coherent torque competes with the antiferromagnetic exchange.

Another mistake appears later in the main text on page 4, right column, second paragraph. The text states that "the components of the spin density along $\mathbf{z}$ and $\mathbf{x}$ result in a strong in-plane coherent torque and weaker out-of-plane exchange torque, respectively." This sentence should read "the components of the spin density along $\mathbf{z}$ and $\mathbf{x}$ result in an out-of-plane torque with a stronger exchange component and a weaker coherent component."

Similarly, the sentence "This additional $\mathbf{y}$ component is expected to produce an additional out-of-plane coherent torque as well as an in-plane exchange torque." should read "This additional $\mathbf{y}$ component is expected to produce a strong in-plane coherent torque and weaker in-plane exchange torque."

The authors thank T. Jungwirth for pointing out these two inconsistencies.

[1] H. B. M. Saidaoui, A. Manchon, and X. Waintal, Phys. Rev. B 89, 174430 (2014).

[2] A. S. Nunez, R. A. Duine, P. Haney, and A. H. MacDonald, Phys. Rev. B 73, 214426 (2006).

[3] H. V. Gomonay and V. M. Loktev, Phys. Rev. B 81, 144427 (2010).

[4] J. Zelezny, H. Gao, K. Vyborny, J. Zemen, J. Masek, A. Manchon, J. Wunderlich, J. Sinova, and T. Jungwirth, Phys. Rev. Lett. 113, 157201 (2014).

[5] T. Jungwirth, X. Marti, P. Wadley, and J. Wunderlich, Nat. Nanotechnol. 11, 231 (2016).

[6] E. V. Gomonay and V. Loktev, Low Temp. Phys. 40, 17 (2014). 